\title{
Progress on PEEM3 - An Aberration Corrected X-Ray Photoemission Electron Microscope at the ALS
}

\author{
A.A.MacDowell ${ }^{1}$, J.Feng ${ }^{1}$, A.DeMello ${ }^{1}$, A.Doran ${ }^{1}$, R.Duarte ${ }^{1}$, E.Forest ${ }^{2}$, N.Kelez ${ }^{1}$, \\ M.A.Marcus ${ }^{1}$, T.Miller ${ }^{1}$, H.A.Padmore ${ }^{1}$, S.Raoux ${ }^{3}$, D.Robin ${ }^{1}$, A.Scholl ${ }^{1}$, \\ R.Schlueter ${ }^{1}$, P .Schmid ${ }^{1}$, J.Stöhr ${ }^{4}$,W.Wan ${ }^{1}$, D.H.Wei ${ }^{5}$ and Y.Wu ${ }^{6}$ \\ ${ }^{1}$ Advanced Light Source, Lawrence Berkeley National Lab. Berkeley, CA 94720, USA \\ ${ }^{2}$ High Energy Accelerator Research Organization, 1-1 Oho, Tsukuba, Ibaraki,305-0810, Japan \\ ${ }^{3}$ IBM, Almaden Research Center, 650 Harry Road, San Jose, CA 95120, USA \\ ${ }^{4}$ Stanford Synchrotron Radiation Laboratory, PO Box 20450, Stanford, CA 94309, USA \\ ${ }^{5}$ NSRRC, 101 Hsin-Ann Road, Hsinchu 30077, Taiwan \\ ${ }^{6}$ Department of Physics, Duke University, Durham, NC 27708, USA
}

\begin{abstract}
A new ultrahigh-resolution photoemission electron microscope called PEEM3 is being developed and built at the Advanced Light Source (ALS). An electron mirror combined with a much-simplified magnetic dipole separator is to be used to provide simultaneous correction of spherical and chromatic aberrations. It is installed on an elliptically polarized undulator (EPU) beamline, and will be operated with very high spatial resolution and high flux to study the composition, structure, electric and magnetic properties of complex materials. The instrument has been designed and is described. The instrumental hardware is being deployed in 2 phases. The first phase is the deployment of a standard PEEM type microscope consisting of the standard linear array of electrostatic electron lenses. The second phase will be the installation of the aberration corrected upgrade to improve resolution and throughput. This paper describes progress as the instrument enters the commissioning part of the first phase.
\end{abstract}

Keywords: Photoemission Electron Microscope, PEEM, Aberration Correction.

PACS: Replace this text with PACS numbers; choose from this list: 07.85.Qe, 07.78.+s

\section{INTRODUCTION}

The photoemission electron microscope (PEEM) has been developed since 1930 [1] to study the surface and thin film properties of various materials. In a PEEM, photons impinging on the sample cause the emission of secondary photoelectrons. These electrons are accelerated to typically $10-30 \mathrm{keV}$ and focused to produce a magnified intermediate image by an immersion objective lens. A series of projection lenses are used to magnify this intermediate image further and form a final image on a CCD or other imaging detector. When tunable X-rays from a storage ring are used, different contrast mechanisms such as topographic, elemental, chemical, orientation and magnetic are available. The lateral resolution limit of state-of-the-art PEEMs is about $20 \mathrm{~nm}$ such as the secondgeneration PEEM2 instrument [2] now operating on a bend magnet beamline at the Advanced Light Source (ALS). This limit is due to the spherical and chromatic aberrations of the immersion objective lens. By incorporating an electron mirror [3] with its own aberrations but of opposite sign, aberration cancellation can be effected with consequent resolution and throughput improvement. The ALS has embarked on the building of such a PEEM3 instrument that will be stationed on a dedicated EPU insertion device at Beamline 11.0.1 [4].

\section{DESCRIPTION OF THE PEEM3 INSTRUMENT}

Figure 1 shows the schematic overview of the electron optics of PEEM3. Photoelectrons from the sample are collected and accelerated by the immersion objective lens to the nominal energy of $20 \mathrm{keV}$. The objective lens is an electrostatic four-electrode lens, in which the sample is also part of the lens and located 2mm away from the second grounded electrode. This distance is adjustable to accommodate different sample cases. This objective lens is 
similar to the PEEM2 objective lens [2], whose electron optical properties have been optimized to have small aberrations [5]. An image with a magnification of 11.23 is formed at the entrance plane of the separator section (indicated by image arrow in Fig.1) between the field lens and the first quadrupole. This field lens makes the field ray parallel upon entering the beam separator. The objective lens, together with the field lens, form a telescopic round lens system. This is necessary for the mirror to run in the so-called symmetric mode in which first-order chromatic distortion and third-order coma can be cancelled [6]. To cope with the inevitable stray DC magnetic fields mechanical misalignments and tolerance errors and we employ dodecapole correctors as indicated in Figure 1 . The choice of dodecapoles was driven by the desire to have not only quadrupole and octapole correctors but also a hexapole component, as three-fold astigmatism has been a problem in PEEM2 which only has octapole correctors. All correctors are dodecapoles for design consistency.

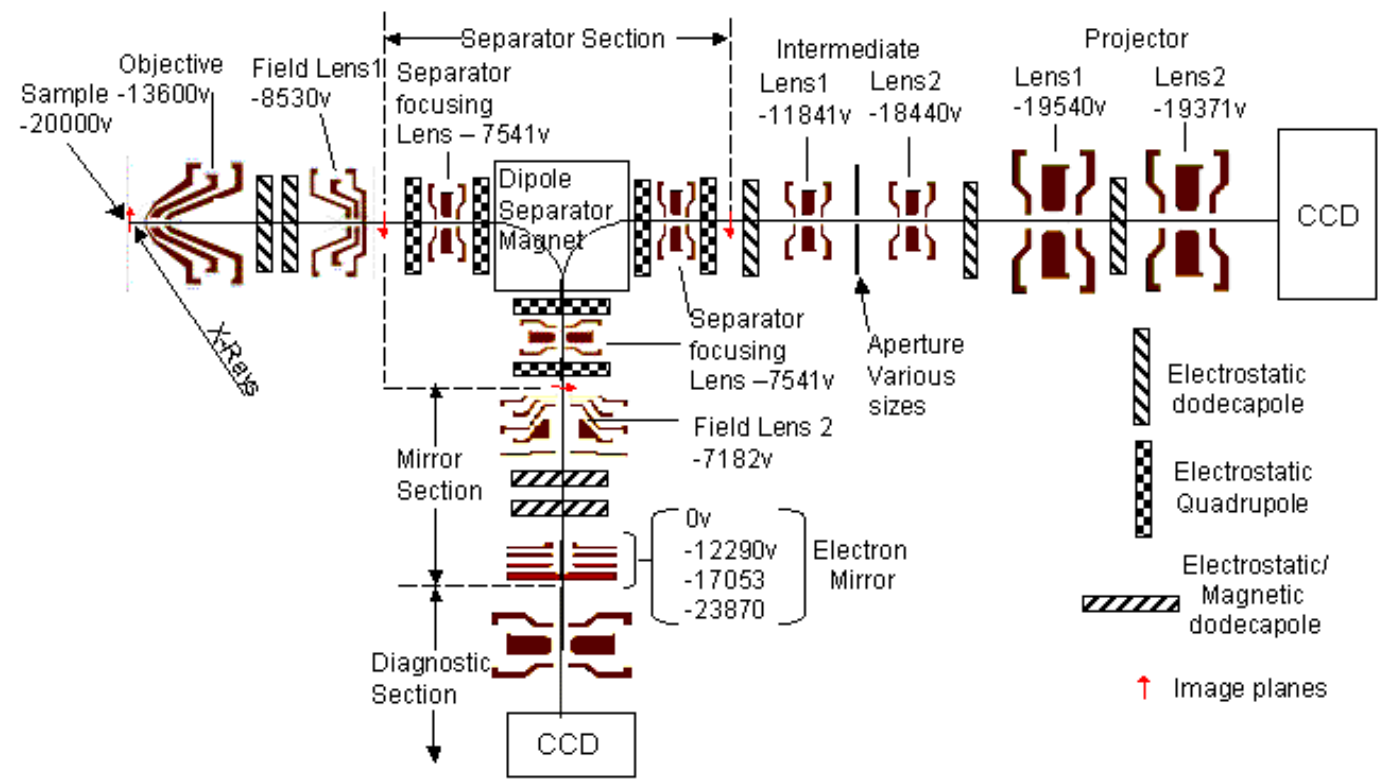

FIGURE 1. Schematic layout of the electrostatic lenses, separator magnet and electron mirror of the PEEM3 microscope. Typical lens voltages are given. As a scale indicator, the distance from the sample to the right hand CCD is 1350mm.

The separator section consists of three Einzel lenses, six quadrupoles and the dipole separator magnet itself [7]. Simple round Einzel lenses were chosen to be the main focusing lenses as the deviation from rotational symmetry caused by the bending magnet and the remaining aberrations of the lenses are small. The weak electrostatic quadrupoles provide the required stigmatic image correction and the 4 degrees of freedom per leg for the electron beam position and angle. On the first pass, the electron beam leaves the separator section chromatically dispersed as it enters the mirror section

The mirror itself consists of four electrodes with the actual mirror reflecting electrode shaped as a spherical segment of radius $5.6 \mathrm{~mm}$. The entrance electrode is at ground voltage, while the potentials of other electrodes give three degrees of freedom to determine the focal length, chromatic aberration and the spherical aberrations of the mirror [8]. In order to cancel coma generated by the mirror, the magnification of the mirror is chosen to be -1 and a field lens is placed near the image plane (separator exit on the first pass) to ensure that the linear optics are telescopic for the fundamental ray trajectories. A pair of magnetic/electrostatic dodecapoles effect adjustments of beam trajectory within the mirror section. The set up of the separator and mirror is not going to be trivial. A projector lens and CCD detector are located behind the mirror, which is to be used as a diagnostic PEEM. This PEEM will allow us to independently test and optimize the first half of the beam separator and the incoming beam at the mirror. In this operational mode, the lens voltages are rearranged and the electron beam passes though a $500 \mu \mathrm{m}$ diameter hole in the reflecting electrode before being projected onto the diagnostic CCD.

The mirror section has magnification of -1 , so the second pass through the separator cancels the chromatic dispersion of the separator on the first pass. This is the new development for these low energy PEEM microscopes. A significant amount of design work was put into developing a separator magnet with the initial work directed along 
the lines of the SMART type separator concept [9]. Here the separator is designed to be aberration free to high order and therefore imposes severe electron optical and engineering challenges. A detailed design was worked out [10] and is very similar to the SMART design being a single magnetic unit wound such that there are 8 dipoles on each pass that employ only edge focusing. The performance of this design depends critically on stringent machining tolerances and is essentially a non-tunable design. By relaxing the chromatic aberration condition for the separator we are now proposing to use, the engineering tolerances are more reasonable and it is also tunable.

The transfer optics and projection system are used to magnify the intermediate image at the exit plane of the magnetic separator onto the CCD detector without distortion. The optical properties of this type of lens with different geometries of individual electrodes have been studied in great detail by Rempfer [11]. Based on this work, the transfer and projection system of PEEM3 consists of four electrostatic uni-potential lenses with the last two lenses having a larger aperture than the first two.

The resolution of the system is dependant on the aberrations of electron optics, the energy and angular spread of the initial electrons and diffraction effects due to the wave nature of the electron. This has been modeled [12] with the resolution defined as the rise-distance of $15 \%$ - $85 \%$ in intensity of the edge scanning the electron point-spread function. The resolution for $100 \%$ transmission is $50 \mathrm{~nm}$ with the mirror corrector - a significant improvement from that of $440 \mathrm{~nm}$ without correction. The best predicted resolution is $5 \mathrm{~nm}$ at $2 \%$ transmission, as opposed to $20 \mathrm{~nm}$ at $0.5 \%$ transmission for PEEM2.

\section{PROGRESS WITH PEEM3 CONSTRUCTION}

The engineering requirements for this instrument are quite demanding. The magnetic shielding requirements are stringent given that the electrons have very low energy at the sample and electron mirror. It is however the long travel length $(1950 \mathrm{~mm})$ of the $20 \mathrm{KeV}$ electrons that drives the specified AC magnetic field down to a required value of $<2 \times 10^{-7}$ Gauss. In the area where the instrument is to be located, AC field magnitudes of $+-2.5 \times 10^{-4}$ Gauss with a time period of $\sim 1 \mathrm{~Hz}$ are measured. The source is most likely the injector booster for the main storage ring. Triple mu-metal shielding is used to reduce the fields to the required level. The entire assembly is to be mounted on a single electron optical table that sits on an epoxy granite table with visco-elastic sheet dampers to reduce floor vibrations. The mu-metal shields and vacuum tank are supported separately and wrap around the electron optical table connected only via vacuum bellows. Ultra High Vacuum (UHV) is required for the sample and electron mirror region to reduce surface contamination. Rather than try to evacuate the entire rather large instrument to UHV we have adopted localized UHV sections with the electrons passing in and out of them via small conductance limited holes. The power supplies that supply voltage to the lenses are required to be very stable. An analysis indicated that the electron optics in regions where the electrons have low energy require <+-2 parts per million (ppm) stability. Power supplies for the sample, objective lens and the mirror voltages have been custom fabricated.

Constraints imposed by the project complexity and funding have required that the instrument be deployed in two stages. The first stage is in the PEEM2 mode. The schematic of the electron optics is shown in Figure 2.

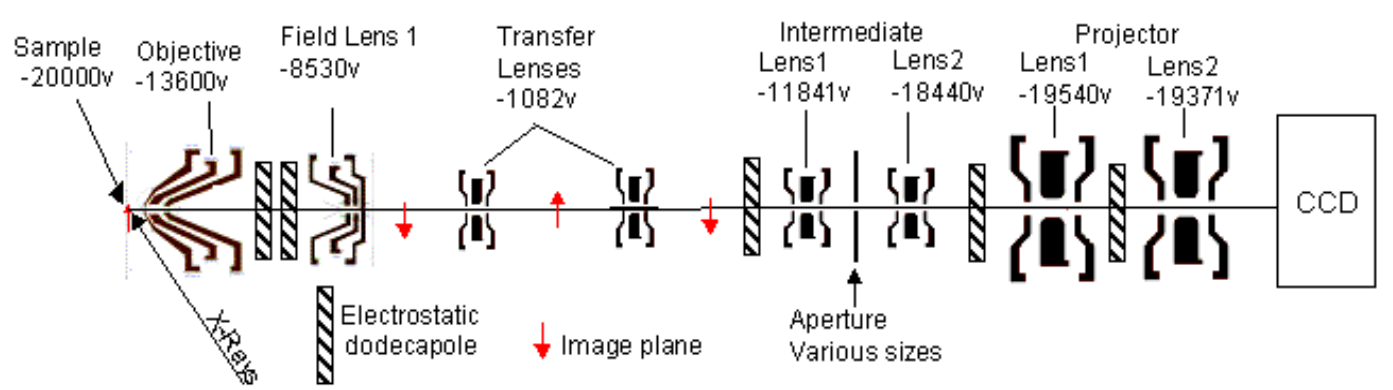

FIGURE 2. Schematic layout of the electrostatic lenses for the PEEM2 (stage 1) mode of the microscope buildup

Here the separator has been replaced with a two-lens (4f) image transfer system. The image from the objective section is relayed to the transfer and projection section. The left panel of Figure 3 shows the instrument undergoing the final assembly. The electron column is visible along with the sample manipulator system mounted in the UHV sub chamber and surrounded by the triple mu-metal shields. The right panel of Figure 3 shows a closer view of the UHV non-magnetic sample manipulator and objective lens column. The manipulator is a novel device based on flexures that allows for 5 degrees of freedom of the sample. The degree of freedom missing is rotation of the sample 
about the electron optic axis, which is not required as the polarization of the x-rays from the EPU can be rotated about this axis. The sample manipulator carries a sample puck that can be inserted and removed via a sample load lock system. The ceramic sample support structure is light and when mounted off the stiff flexures has natural vibration frequencies $>200 \mathrm{~Hz}$ which is high and will help reduce vibration issues. The sample manipulator is mounted on the electron optical bench and provides the required stiff mechanical loop between the sample and objective lens to reduce sample vibration and drift issues. The manipulator's 5 degrees of freedom are driven by non magnetic UHV slides with piezoelectric Nanomotors encoded to 20nm [13]. The stage is designed to be capable of delivering temperature ranges of $60 \mathrm{~K} 1300 \mathrm{~K}$ to the sample. Commissioning of the instrument and connection to the EPU beamline [4] is underway. The current PEEM2 science program will migrate to this instrument while the separator and mirror section is completed offline. This section should be installed in 2007.
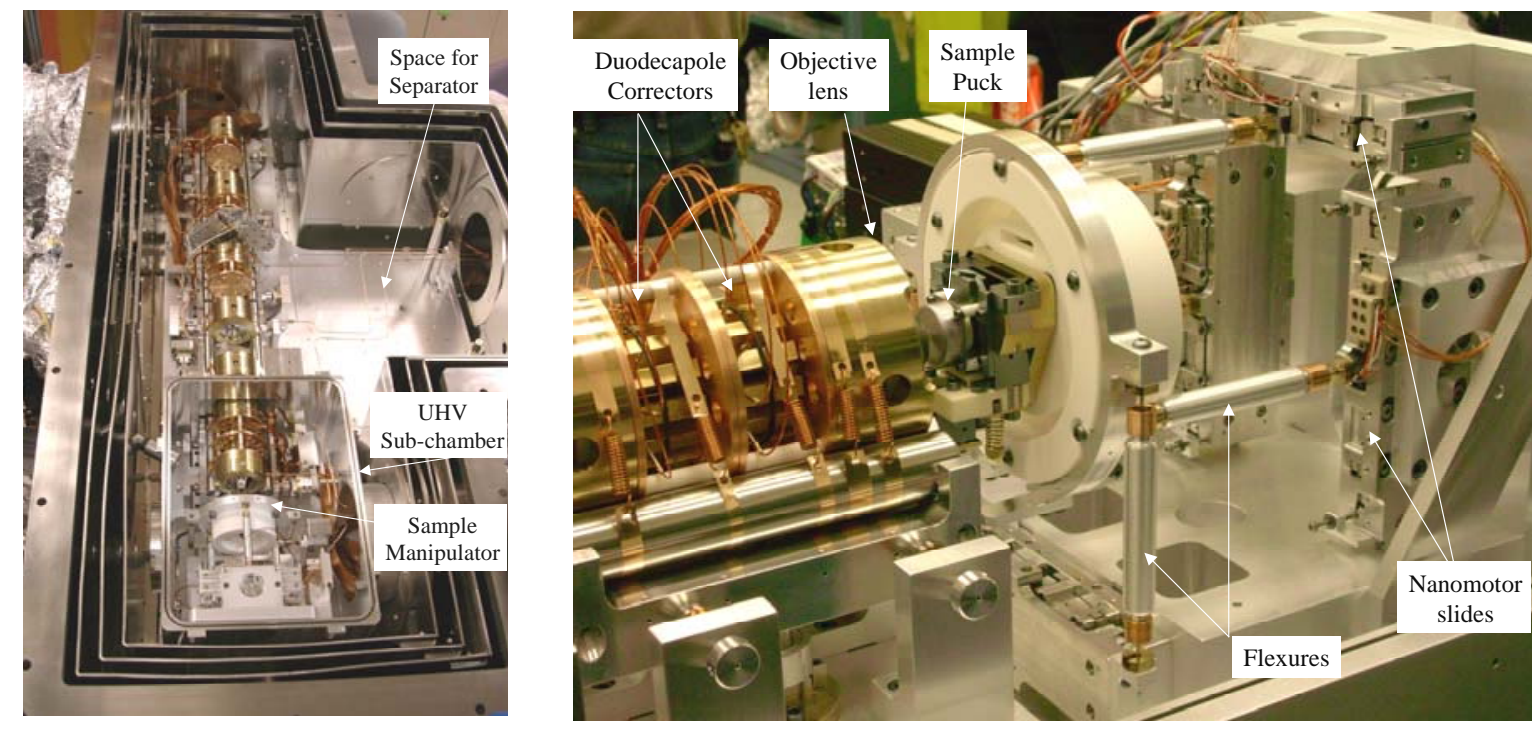

FIGURE 3. Left - the PEEM2 mode electron column and sample manipulator mounted in the vacuum chamber lined with 3 layers of mu-metal. Right - the objective lens, dodecapole correctors and sample puck mounted on the ceramic sample manipulator.

\section{ACKNOWLEDGMENTS}

This work was supported by the Director, Office of Energy Research, Office of Science of the U.S. Department of Energy, under Contract No. DE-AC03-76SF00098.

\section{REFERENCES}

1. E.Brucher, Z. Phys., 86, 448- (1933).

2. S. Anders et al., Rev. Sci. Instrum, 70, 3973-3981(1999).

3. D.Preikszas and H.Rose, J.Electro. Microscopy, 1, 1-9 (1997).

4. T.Warwick et al., “A New VLS grating monochromator beamline with energy stabilization” these proceedings.

5. R.N.Watts, et al., Rev.Sci.Instrum., 68, 3464-3476 (1997).

6. H.Rose and D.Preikszas, Optik, 92, 31-44 (1992).

7. W. Wan, J. Feng and H.A. Padmore , “A new separator design for aberration corrected photoemission electron microscopes “, Nucl. Instr. and Meth. A in press.

8. W.Wan, et al., Nucl.Instr. Meths., A519, 222 -229(2004).

9. R.Fink et.al., J.Elec.Spec Rel Phenom., 84, 231-250 (1997).

10. Y.K.Wu et al., Nucl.Instr. Meths, A519, 230-241 (2004).

11. G.F.Rempfer et al., Ultramicroscopy, 36, 196-221 (1991).

12. J.Feng, et al., J.Phys: 17, S1339-S1350(2005).

13. Ibex Engineering, Newbury Park, CA, 91320, USA 